\title{
Code development for ITER edge modelling - SOLPS5.1
}

\author{
X. Bonnin ${ }^{\text {a, }}$, A.S. Kukushkin ${ }^{\text {b }}$, D.P. Coster ${ }^{c}$ \\ ${ }^{a}$ LIMHP, Université Paris 13, CNRS, Institut Galilée, F-93430 Villetaneuse, France \\ ${ }^{b}$ ITER Organization, Cadarache Centre, F-13108 Saint-Paul-lèz-Durance, France \\ ${ }^{c}$ Max-Planck-Institut für Plasmaphysik, D-85748 Garching-bei-München, Germany
}

\begin{abstract}
Most ITER divertor modelling work to date used the B2-Eirene (SOLPS4) code package, coupling a 2D fluid description of the charged plasma species (B2) to a Monte-Carlo kinetic description of the neutrals (Eirene). In recent years, the emphasis at ITER has been on completing the neutral model, including neutral-neutral collisions, opacity effects, radiation transport, etc. Elsewhere, new physics, numerics, and algorithmic improvements, such as $\mathbf{E} \times \mathbf{B}$ and diamagnetic drifts, electric currents, ion and neutral heat and particle flux limits, wall material mixing and surface temperature evolution, and bundling of heavy ions species, as well as switching to cell-centred velocities and using an internal energy instead of a total energy equation, gave birth to the B2.5 code, combined with Eirene as SOLPS5. We report on work in progress to merge these advances with the ITER-specific model of the edge and divertor.
\end{abstract}

JNM Keywords: Mathematical and Computational Methods, Theory and Modelling PSI-18 Keywords: B2/EIRENE, Divertor modelling, Edge modelling, ITER PACS: 52.40.Hf, 52.65.-y

*Corresponding author address: LIMHP, Université Paris 13, CNRS; Institut Galilée, 99 avenue Jean-Baptiste Clément, F-93430 Villetaneuse, France 
*Corresponding author e-mail: bonnin@limhp.univ-paris13.fr

Presenting author: Xavier Bonnin

Presenting authore-mail: bonnin@limhp.univ-paris13.fr

\section{Introduction}

In this paper we review the current status of the SOLPS (Scrape-Off Layer Plasma Simulation) suite of codes, also referred to as B2-Eirene, which is being used for the design and modelling of the ITER edge plasma and divertor. We begin by detailing the different versions of SOLPS that are currently in use. We then compare output between the two "bestphysics" models, SOLPS4.2 [1] and SOLPS5.1. After identifying the principal sources of difference between the two code versions, we quantify their impact on the ITER edge solution. We then list briefly some other aspects of edge modelling physics that need to be better treated in such codes and discuss how the SOLPS suite is being modified to handle these challenges.

\section{Description of the various SOLPS versions}

Historically, the SOLPS suite of codes [2] has grown for the coupling of a plasma fluid solver, B2 and then later B2.5 [3,4], to a Monte-Carlo transport code for neutrals, Eirene [5], as well as several pre- and post-processing utilities. Since the two main components are maintained and developed by different teams, various "weddings" have taken place between the two families leading to a multiplicity of SOLPS versions. These are summarized in Table I. Eirene code development, done at FZ Jülich, has yielded several generations of the code. Distributed as part of the standard SOLPS4.0 [6] and SOLPS5.0 are so-called "old" and “new" versions, corresponding to Eirene as of 1996 and 1999 respectively. Users may then choose within their local installation which version they prefer. Eirene-99 includes more detailed atomic and molecular physics treatment than its predecessor, as well as an improved 
description of wall reflection models, among other features. Afterwards, a significant coding effort has gone into providing much better numerics, including dynamic allocation, use of Fortran-95 standards and including new physics such as photon transport and opacity effects, neutral-neutral collisions and better treatment of elastic collisions [1]. Many of these developments were driven by the physics needs of the ITER team and this Eirene "face-lift" version was then coupled with B2, with some ITER-specific improvements [7] concerning particle balance in particular, to produce SOLPS4.2, which is the version used by the ITER team in the last four years. The Eirene group has just finished preparing a parallelized version of Eirene, coupled to the ITER version of B2 to form SOLPS4.3, the current ITER version of the code.

In parallel, the fluid plasma code was entirely rewritten as B2.5. Numerically, this rewriting involved dynamic memory allocation, indirect addressing of neighbours (to handle multiple topologies), and moving to a non-staggered grid. On the physics side, improvements included drifts and currents, and the replacement of the ion total energy equation by an ion internal energy equation. Other code developments are also listed in Section 4 below. Coupling B2.5 to the older Eirene versions formed SOLPS5.0. The current paper reports on the current attempts to couple the Eirene "face-lift" (and eventually the parallelized Eirene version) with B2.5, which has been dubbed SOLPS5.1. All the latest developments to SOLPS5.0 are carried into SOLPS5.1 as they are tested and validated. However, the numerics of the drifts in SOLPS5.0 are not yet entirely satisfactory, and therefore some effort has been devoted to improving the code stability and domain of convergence. This is being reported in [8], again with coupling to more recent Eirene versions envisaged for the future. Lastly, B2.5 has also been rewritten to take advantage of adaptive meshing schemes, in view of coupling it to 1-D transport codes and better solution of time-dependent travelling events such as ionisation fronts [9]. This forms the basis for SOLPS6.0, but there coupling to recent 
Eirene versions has not yet been thought through. With this context in place, we now move to a more detailed comparison of the output of ITER runs between SOLPS4.2 and SOLPS5.1, i.e. combining the currently best available models for the plasma edge.

\section{Comparisons between SOLPS4.2 and SOLPS5.1 for ITER}

The methodology used for comparing output from both codes has been to rely on a wellconverged SOLPS4.2 case as the baseline, and then attempt to continue this case with SOLPS5.1. As differences are identified, the SOLPS5.1 code is made "backwardcompatible", in the sense that options are added to reproduce the old numerical treatment. The new code is then re-run and the new differences assessed. We illustrate this process by plotting the poloidal particle and energy fluxes past the divertor throat (Figure 1) and reaching the targets (Figure 2) for the various changes made as the benchmark progresses. Radial fluxes show similar behaviour but the differences there are smaller.

Such careful benchmark exercises usually reveal some subtle coding errors and this time was no exception. Among the first discrepancies spotted was the calculation of cell volumes, and cross-sectional areas of flux tubes for fluxes computations, both of which had to be recoded ("Perp. area") and their consequences carried through ("Consistent geometry"). In the closed field lines region, the flux limiters formulations were different ("Luciani flux limits"). SOLPS4.x classical transport relies on the Braginskii formalism, while SOLPS5.x uses the Balescu formulation, which is appropriate when ambipolarity is no longer assumed ("Ion \& el. classical transport"). SOLPS4.x used a modified upwind discretisation scheme, while SOLPS5.x used the hybrid scheme due to Patankar ("Upwind scheme"). Several currents contributions needed to be turned off in SOLPS5.x ("No currents" and "Particle fluxes") and the electric potential set to $3.1 T_{e}$ ("Potential equation"). SOLPS4.x did not include ion flux limits ("No ion flux limits"), but its ion energy equation contains a kinetic energy flux term 
("Kinetic energy flux"). Lastly, the viscosity flux limits were coded quite differently in the two versions ("Viscosity limit").

In addition to these points, a few "irreconcilable" differences remain. SOLPS4.x versions use a staggered grid, solving for the poloidal velocity on cell faces, while SOLPS5.x versions use a non-staggered grid with cell-centred parallel velocities. Thus, the comparison between the two code outputs always keeps an element of velocity interpolation. More importantly, SOLPS4.x uses a total energy equation to solve for the ion temperature, while SOLPS5.x relies on an internal energy formulation. This means that naïvely comparing "ion heat fluxes" can be misleading, as both codes understand this term to signify a slightly different quantity.

After all the above, the differences in the divertor throat fluxes were brought to the percent level, which is excellent agreement, but the situation at the divertor targets remains a concern, as differences there remain in the 50\% range for particle and ion energy fluxes. More work needs to be done to understand these discrepancies better and resolve them. It is noteworthy that, apart from corrections to some geometrical factors which had a large impact near the Xpoint (where the grid is furthest from orthogonality) and at the targets, the main contribution to the difference came from the ambipolarity assumption. This suggests that new ITER solutions with non-zero currents may well change noticeably from previous current-free calculations.

\section{Progress on some further developments for SOLPS}

In addition to the comparison work between the already existing versions of the code, several other issues of critical need for ITER and current devices are being addressed. In particular, the issue of high- $\mathrm{Z}$ ion sequences such as tungsten, the occurrence of wall material mixing, the time evolution of wall surface temperature and its impact of wall erosion processes, as well as hydrogen inventory and tritium retention, need to be addressed in a selfconsistent edge model of the plasma in the device. We briefly describe some avenues of code 
development to that effect below. Other developments, such as improved numerical drifts treatment [8], coupling to the ASPOEL code for description of the far SOL [10] and parallelisation of Eirene, are the topic of accompanying papers.

\section{Full tungsten treatment}

Under normal circumstances, the fluid plasma treatment of the plasma equations requires that the entire isonuclear sequence of a given species be treated simultaneously. For the case of tungsten, this means treating a set of 75 additional equations. Although B2.5 is not in principle limited in terms of the number of species treated, memory requirements and execution time can become an issue for high- $Z$ cases. Some effort has thus been spent in making sure that the code could indeed run with the full implement of ITER species: $(2 \mathrm{D}+2$ $\mathrm{T}+3 \mathrm{He}+5 \mathrm{Be}+7 \mathrm{C}+75 \mathrm{~W}=94$ species) and in speeding up the numerics related to the computation of the matrix elements. Apart for the impediment of long run times, such runs also require the availability of reliable atomic data for tungsten. This data is currently being calculated [11] by a joint effort between the ASDEX-Upgrade and ADAS teams. Also missing is sputtering data for combinations involving incident ions from a wall material impinging on a different material. Computation of theoretical yields using SDTrimSP is under way at IPP-Greifswald. Additionally, particularly for material mixes with large mass mismatches such as $\mathrm{W}$ in $\mathrm{C}$, the sputtering yield of an ion incident on a W/C mixture is not a linear combination of its sputtering yields on atomic $\mathrm{C}$ and $\mathrm{W}$ surfaces [12]. This is further complicated by issues of preferential sputtering, where experiments are scarce and yield contradicting information.

\section{Bundled charge states}

Another option for dealing with high- $\mathrm{Z}$ ion sequence is to allow for several of the ions to be bundled into a single plasma "species". SOLPS5.0 and 5.1 have been made compatible with such a bundled charge state scheme, basically by allowing the average charge, square 
charge, and ionisation potential of each species to vary according to local electron density and temperature. The burden now falls on the A\&M data community to provide data for different bundling scenarios of heavy ion species. A test case using D + Ar in a JET geometry has shown that the plasma solution was little affected by relatively strong bundling (halving the total number of species treated) unless the solution neared the detachment transition. However, the collisional-radiative model for each bundled species currently does not include the effect of charge-exchange reactions which do not move an ion outside of its bundle. Doing so would require making the model dependent on local neutral density as well.

\section{Wall temperature model and mixed materials}

The ITER wall also presents a modelling challenge because of its multi-material nature. It is important to follow the erosion and redeposition of wall materials, on wall elements of both similar and dissimilar composition. Moreover, it is necessary to follow the composition of surface layers that may form, as well as the time evolution of the surface temperature, to be able to determine how erosion rates will evolve in time. It is also important to take into account, in addition to the temperature and flux dependencies of erosion processes, chemical effects coming from the partial coverage of the bulk wall element surface with re-deposited material of a different nature, which may strongly impede chemical erosion, but are much less well understood. In SOLPS4.2, on the Eirene side, it is already possible to distinguish between erosion-dominated and deposition-dominated wall elements, whilst in B2.5 the temperature evolution and surface layer composition can be followed in detail [12]. Transferring this capability to Eirene remains to be done.

\section{Hydrogen inventory and tritium retention}

Another essential issue for ITER is hydrogen inventory and tritium retention in walls. Correct treatment of this physics requires a multi-scales approach, from hopping of hydrogen among lattice sites, to transport along grain boundaries, and bulk diffusion, in addition to the 
plasma recycling-erosion-transport-redeposition cycles. Implementation of a hierarchy of models [13] to encompass these phenomena is currently in its build-up stages.

\section{Conclusions and outlook}

We have presented in this paper the current status of the merging of the best ITER-specific physics model for the plasma edge, embodied in SOLPS4.2, with the latest plasma fluid treatment from B2.5, into SOLPS5.1. The two codes still harbour important differences in their results, notably for ion particle and energy fluxes at the targets. Some of these differences are attributable to differing numerical choices with other remaining ones have less clear of an origin. The ambipolarity assumption in SOLPS4.2 is seen to lead to the most significant of the physics-based differences. Besides this comparison effort, we have reviewed several avenues of ongoing code development aimed at improving the predictive capabilities of SOLPS for ITER, such as treatment of high-Z ions, transport and redeposition of erosion products and their consequences, including hydrogen inventory and tritium retention.

\section{Acknowledgements}

This work has been undertaken as part of contract ITER/CT/07/346. The authors also would like to thank V. Rozhansky for fruitful discussions.

\section{References}

[1] V. Kotov et al., Contrib. Plasma Phys. 46 (2006) 635.

[2] R. Schneider et al., Contrib. Plasma Physics 46 (2006) 3.

[3] B.J. Braams, Contrib. Plasma Phys. 36 (1996) 276.

[4] V. Rozhansky et al., Nuclear Fusion 41 (2001) 387.

[5] D. Reiter, J. Nucl. Mater. 196-198 (1992) 80.

[6] D.P. Coster et al., Proc. $19^{\text {th }}$ Fusion Energy Conference, Lyon, 2002, paper IAEA-CN94/TH/P2-13M.

[7] A.S. Kukushkin et al., Plasma Phys: Controlled Fusion 44 (2002) 931. 
[8] V. Rozhansky et al., these proceedings, paper P2-78.

[9] X. Bonnin et al., J. Nucl. Mater. 313-316 (2003) 909.

[10] F. Subba et al., these proceedings, paper P2-31.

[11] T. Pütterich et al., Plasma Phys. Controlled Fusion, to be published.

[12] D.P. Coster et al., Physica Scripta T124 (2006) 9.

[13] X. Bonnin et al., 34 ${ }^{\text {th }}$ EPS, Europhysics Conference Abstracts 31F P4.036. 


\section{Table captions}

Table I: Summary of the various SOLPS versions according to their plasma fluid component (B2, B2.5) and kinetic neutral treatment (Eirene). Arrows indicate future planned couplings. 


\begin{tabular}{|c|c|c|c|c|}
\hline $\begin{array}{c}\ddots \text { Kinetic neutrals } \\
\text { treatment } \\
\text { Fluid plasma' } \because \\
\text { treatment }\end{array}$ & Eirene-96 & Eirene-99 & Eirene « facelift » & $\begin{array}{c}\text { Parallelized } \\
\text { Eirene }\end{array}$ \\
\hline $\mathrm{B} 2[2]$ & SOLPS4.0 [6] & $\begin{array}{c}\text { SOLPS4.2 [7] } \\
\text { (ITER-optimised version) }\end{array}$ & SOLPS4.3 \\
\hline B2.5[3,4] & SOLPS5.0 [2] & $\begin{array}{c}\text { SOLPS5.1 } \\
\text { (this work) }\end{array}$ & \\
\hline $\begin{array}{c}\text { Improved drifts } \\
\text { treatment }\end{array}$ & SOLPS5.2[9] & & \\
\hline $\begin{array}{c}\text { Adaptive } \\
\text { meshes }\end{array}$ & SOLPS6.0 [10] & & \\
\hline
\end{tabular}

Table I 


\section{Figure captions}

Figure 1: Comparison of the integrated particle and energy fluxes at the inner and outer divertor throat entrances of ITER for various implementations of the SOLPS code.

Figure 2: Comparison of the integrated particle and energy fluxes at the inner and outer divertor targets of ITER for various implementations of the SOLPS code. 


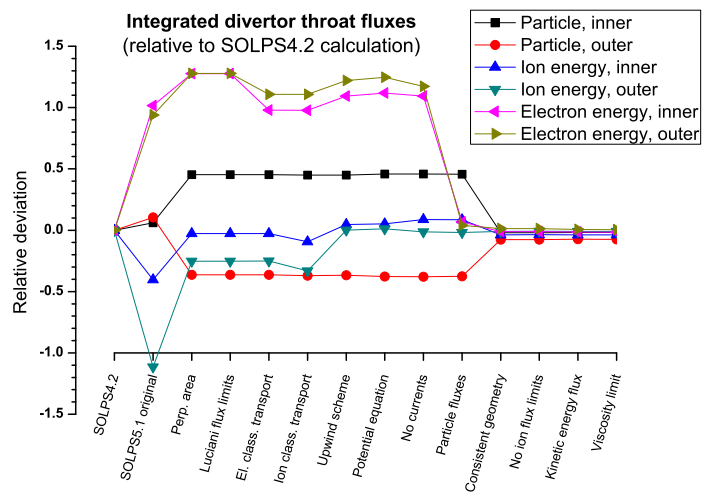

Figure 1. 


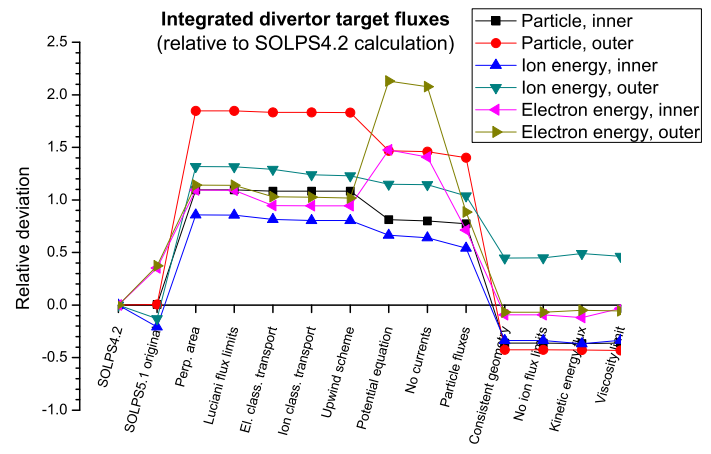

Figure 2. 